5-1-2009

\title{
Covariate-Adjusted Constrained Bayes Predictions of Random Intercepts and Slopes. Sujit Ghosh is a
}

Robert H. Lyles

EmoryUniversity, rlyles@emory.edu

Reneé H. Moore

University of Pennsylvania, rhmoore@mail.med.upenn.edu

Amita K. Manatunga

EmoryUniversity, amanatu@emory.edu

Kirk A. Easley

Emory University, keasle2@emory.edu

Follow this and additional works at: http://digitalcommons.wayne.edu/jmasm

Part of the Applied Statistics Commons, Social and Behavioral Sciences Commons, and the Statistical Theory Commons

\section{Recommended Citation}

Lyles, Robert H.; Moore, Reneé H.; Manatunga, Amita K.; and Easley, Kirk A. (2009) "Covariate-Adjusted Constrained Bayes Predictions of Random Intercepts and Slopes. Sujit Ghosh is a," Journal of Modern Applied Statistical Methods: Vol. 8 : Iss. 1 , Article 7. DOI: $10.22237 /$ jmasm/1241136360

Available at: http://digitalcommons.wayne.edu/jmasm/vol8/iss1/7

This Regular Article is brought to you for free and open access by the Open Access Journals at DigitalCommons@WayneState. It has been accepted for inclusion in Journal of Modern Applied Statistical Methods by an authorized editor of DigitalCommons@WayneState. 


\title{
Covariate-Adjusted Constrained Bayes Predictions of Random Intercepts and Slopes
}

\author{
Robert H. Lyles \\ Reneé H. Moore \\ Emory University \\ University of Pennsylvania \\ Amita K. Manatunga \\ Emory University \\ Kirk A. Easley \\ Emory University
}

Constrained Bayes methodology represents an alternative to the posterior mean (empirical Bayes) method commonly used to produce random effect predictions under mixed linear models. The general constrained Bayes methodology of Ghosh (1992) is compared to a direct implementation of constraints, and it is suggested that the former approach could feasibly be incorporated into commercial mixed model software. Simulation studies and a real-data example illustrate the main points and support the conclusions.

Key words: Mixed linear model, prediction, random effects, shrinkage.

\section{Introduction}

The standard mixed linear model (e.g., Laird \& Ware, 1982) remains a popular practical tool for analyzing longitudinal, repeated measures, or otherwise correlated continuous data. In such analyses, the prediction of linear combinations of fixed and random effects can be of great interest. The typical approach implemented in commercial software is to obtain empirical best linear unbiased predictors (EBLUPs), which estimate the posterior mean of the linear combination given the response data (Littell, et al., 2006). The general acceptance of these empirical Bayes-like predictions stems from their intuitive appeal and their theoretical

Robert H. Lyles is an Associate Professor in the Department of Biostatistics at The Rollins School of Public Health. Email: rlyles@emory.edu. Reneé Moore is an Assistant Professor in the Department of Biostatistics and Epidemiology in the School of Medicine. Email: rhmoore@mail.med.upenn.edu. Amita Manatunga is a Professor in the Department of Biostatistics at The Rollins School of Public Health. Email: amanatu@emory.edu. Kirk Easley is a Senior Associate in the Department of Biostatistics at The Rollins School of Public Health. Email: keasle2@emory.edu. underpinnings as minimal prediction mean squared error estimates (Searle, et al., 1992). They are also referred to as shrinkage estimators, given their characteristic of pulling subject-specific predictions toward a population mean.

Due to the shrinkage phenomenon, EBLUPs stemming from linear mixed models exhibit distributions that can be much narrower than those assumed to characterize the random variables being predicted. Several authors (e.g., Efron \& Morris, 1971; Louis, 1984; Ghosh, 1992) have suggested potential drawbacks to this general feature and proposed methods that reduce shrinkage and/or more closely match the predictor and underlying true distributions.

One effect of overshrinkage in certain applications is that it can lead to a lack of sensitivity for identifying extreme experimental units relative to a fixed threshold (i.e., the probability that an EBLUP lies beyond a threshold given that the true random variable does can be quite small). To improve sensitivity in such a context, Lyles and Xu (1999) proposed constrained Bayes predictors of random intercepts and slopes aimed to minimize mean squared error of prediction (MSEP) given that the means and variances of the predictor distributions match those of the true random effects. Lyles, et al. (2007) introduced additional prediction criteria (e.g., regional bias and 


\section{BAYES PREDICTIONS OF RANDOM INTERCEPTS AND SLOPES}

MSEP) that are relevant when extreme subjects are of key interest and they suggested that the constrained Bayes approach can be an appealing alternative in such situations. Constrained Bayes prediction of random effects has not been widely advocated for use in the mixed linear model context.

The models considered by Lyles and $\mathrm{Xu}$ (1999) are extended here to use fixed and/or time-dependent covariates, and their direct constrained Bayes strategy is compared with the general paradigm advocated by Ghosh (1992). This comparison is relevant for two reasons.

First, while the criteria put forth by Lyles and $\mathrm{Xu}$ are specific to the mixed linear model, Ghosh's approach originates from a more general and decidedly Bayesian point of view. Ghosh provides a paradigm for minimizing a mean squared error criterion subject to matching the posterior expectation of the first two moments of a parameter distribution to corresponding moments of the histogram of the set of estimates. It is therefore useful to assess the performance of Ghosh's paradigm in the mixed model setting and to compare it against an approach that is directly rooted in that context.

Second, Ghosh's method is general, flexible, and implemented in a straightforward and consistent manner. Therefore its validation against an approach directly rooted in the mixed model setting could highlight, for practitioners and commercial mixed linear model software developers, the viability of an accessible alternative prediction method.

\section{Methodology}

\section{Models and Posterior Mean Predictions}

Two familiar normal-theory mixed linear models are used for illustration: the random intercept and random intercept/slope models, respectively.

The random intercept (or one-way random effects ANOVA) model is specified as follows (e.g., Searle, et al., 1992):

$$
Y_{i j}=\mu+b_{i}+e_{i j}
$$

$\left(i=1,2, \ldots, k ; j=1,2, \ldots, n_{i}\right)$, with $i$ indexing the subject and $\mathrm{j}$ indexing the observation. Typical normality assumptions dictate that $\mathrm{b}_{\mathrm{i}} \sim \mathrm{N}\left(0, \sigma_{\mathrm{b}}^{2}\right)$ and $\mathrm{e}_{\mathrm{ij}} \sim \mathrm{N}\left(0, \sigma_{\mathrm{W}}^{2}\right)$, with independence across subjects and between the random terms $b_{i}$ and $e_{i j}$.

Under model (1), a common objective is to predict the $\mathrm{i}^{\text {th }}$ subject's random subjectspecific mean, i.e., $\mu_{i}=\mu+b_{i}(i=1, \ldots, k)$. The EBLUP, as provided by standard mixed model software, is an estimate of the posterior mean $\mathrm{E}\left(\mu_{\mathrm{i}} \mid \mathbf{Y}\right)=\mathrm{E}\left(\mu_{\mathrm{i}} \mid \mathbf{Y}_{\mathrm{i}}\right)$, where $\mathbf{Y}$ and $\mathbf{Y}_{\mathrm{i}}$ denote the complete and $i^{\text {th }}$ subject-specific data vectors, respectively:

$$
\begin{gathered}
\tilde{\mu}_{\mathrm{i}}=\mathrm{E}\left(\mu_{\mathrm{i}} \mid \mathbf{Y}_{\mathrm{i}}=\mathbf{y}_{\mathrm{i}}\right)=v_{\mathrm{i}} \overline{\mathrm{y}}_{\mathrm{i}}+\left(1-v_{\mathrm{i}}\right) \mu \\
\text { where } \bar{y}_{\mathrm{i}}=\mathrm{n}_{\mathrm{i}}^{-1} \sum_{\mathrm{j}=1}^{\mathrm{n}_{\mathrm{i}}} \mathrm{y}_{\mathrm{ij}} \text {, and } \\
v_{\mathrm{i}}=\left\{1+\sigma_{\mathrm{w}}^{2} /\left(\mathrm{n}_{\mathrm{i}} \sigma_{\mathrm{b}}^{2}\right)\right\}^{-1} .
\end{gathered}
$$

The parameter $v_{i}$ governs the extent to which the predicted value shrinks toward the population mean $\mu$, with more excessive shrinkage occurring when $v_{\mathrm{i}}$ is small (i.e., when $\sigma_{\mathrm{W}}^{2} /\left(\mathrm{n}_{\mathrm{i}} \sigma_{\mathrm{b}}^{2}\right)$ is large $)$. The BLUP is obtained by replacing $\mu$ in (2) by its best linear unbiased estimate (Searle, et al., 1992), whereas in practice the EBLUP also replaces the variance components in (2) by their estimates.

Next, consider the random intercept/ slope model, also known as a randomized regression or linear growth curve model (e.g., Diggle, et al., 1994):

$$
Y_{i j}=\left(\alpha+a_{i}\right)+\left(\beta+b_{i}\right) t_{i j}+e_{i j}
$$

$\left(i=1,2, \ldots, k ; j=1,2, \ldots, n_{i}\right)$, where $t_{i j}$ denotes the time at which $\mathrm{Y}_{\mathrm{ij}}$ is measured. Typically this model assumes independence across subjects and normally distributed random effects as follows: 


\section{LYLES, MOORE, MANATUNGA \& EASLEY}

$$
\left(\begin{array}{l}
a_{i} \\
b_{i} \\
e_{i j}
\end{array}\right) \sim N_{3}\left\{\left(\begin{array}{l}
0 \\
0 \\
0
\end{array}\right),\left(\begin{array}{ccc}
\sigma_{1}^{2} & \sigma_{12} & 0 \\
\sigma_{12} & \sigma_{2}^{2} & 0 \\
0 & 0 & \sigma^{2}
\end{array}\right)\right\},
$$

with $\sigma_{1}^{2}, \sigma_{2}^{2}, \quad \sigma_{12}$, and $\sigma^{2}$ denoting the variances of the subject-specific intercept and slope deviations, their covariance, and the random error variance, respectively.

Under model (3), it is common to seek predictions of the $i^{\text {th }}$ subject's random intercept $\left(\alpha_{i}=\alpha+a_{i}\right)$ and slope $\left(\beta_{i}=\beta+b_{i}\right)$. As with model (1) and most feasible mixed linear models, standard software provides EBLUPs for these quantities. In this case, they are estimates of the posterior means $\mathrm{E}\left(\alpha_{\mathrm{i}} \mid \mathbf{Y}\right)=\mathrm{E}\left(\alpha_{\mathrm{i}} \mid \mathbf{Y}_{\mathrm{i}}\right)$ and $\mathrm{E}\left(\beta_{\mathrm{i}} \mid \mathbf{Y}\right)=\mathrm{E}\left(\beta_{\mathrm{i}} \mid \mathbf{Y}_{\mathrm{i}}\right)$. The normality assumptions accompanying model (3) yield

$$
\begin{aligned}
\tilde{\beta}_{\mathrm{i}} & =\mathrm{E}\left(\beta_{\mathrm{i}} \mid \mathbf{Y}_{\mathrm{i}}=\mathbf{y}_{\mathrm{i}}\right) \\
& =\beta+\left(\sigma_{12} \mathbf{1}_{\mathrm{n}_{\mathrm{i}}}^{\prime}+\sigma_{2}^{2} \mathbf{t}_{\mathrm{i}}^{\prime}\right) \Sigma_{\mathrm{i}}^{-1}\left(\mathbf{y}_{\mathrm{i}}-\alpha \mathbf{1}_{\mathrm{n}_{\mathrm{i}}}-\beta \mathbf{t}_{\mathrm{i}}\right)
\end{aligned}
$$

where $\boldsymbol{\Sigma}_{\mathrm{i}}=\operatorname{Var}\left(\mathbf{Y}_{\mathrm{i}}\right)=\mathbf{Z}_{\mathrm{i}} \Delta \mathbf{Z}_{\mathrm{i}}^{\prime}+\sigma^{2} \mathbf{I}_{\mathrm{n}_{\mathrm{i}}}, \mathbf{Z}_{\mathrm{i}}$ is the design matrix for the simple linear regression of $\mathbf{Y}_{\mathrm{i}}$ on time $\left(\mathbf{t}_{\mathrm{i}}\right)$ for subject $\mathrm{i}$, and $\Delta=$ Var $\left(a_{i}, b_{i}\right)^{\prime}$. Assuming $n_{i} \geq 2$, Lyles and $\mathrm{Xu}$ (1999) showed that $\mathrm{E}\left(\beta_{\mathrm{i}} \mid \mathbf{Y}_{\mathrm{i}}\right)$ takes an appealing form:

$$
\begin{aligned}
\tilde{\beta}_{\mathrm{i}} & =\mathrm{E}\left(\beta_{\mathrm{i}} \mid \mathbf{Y}_{\mathrm{i}}\right) \\
& =\gamma_{\mathrm{i} 1}+\gamma_{\mathrm{i} 2} \hat{\alpha}_{\mathrm{i}, \mathrm{ols}}+\gamma_{\mathrm{i} 3} \hat{\beta}_{\mathrm{i}, \mathrm{ols}}
\end{aligned}
$$

where $\hat{\alpha}_{\mathrm{i}, \mathrm{ols}}$ and $\hat{\beta}_{\mathrm{i} \text {,ols }}$ represent the ordinary least squares (OLS) intercept and slope from regressing $\mathbf{Y}_{\mathrm{i}}$ on $\mathbf{t}_{\mathrm{i}}$. The coefficients in (5) are given by:

$$
\begin{aligned}
& \gamma_{\mathrm{i} 2}=\left(\sigma_{12} \mathrm{v}_{\beta \mathrm{i}}-\sigma_{2}^{2} \mathrm{c}_{\alpha \beta \mathrm{i}}\right) / \delta_{\mathrm{i}}, \\
& \gamma_{\mathrm{i} 3}=\left(\sigma_{2}^{2} \mathrm{v}_{\alpha \mathrm{i}}-\sigma_{12} \mathrm{c}_{\alpha \beta \mathrm{i}}\right) / \delta_{\mathrm{i}},
\end{aligned}
$$

and

with

$$
\gamma_{\mathrm{i} 1}=\beta\left(1-\gamma_{\mathrm{i} 3}\right)-\alpha \gamma_{\mathrm{i} 2},
$$

$$
\delta_{\mathrm{i}}=\left(\mathrm{v}_{\alpha \mathrm{i}} \mathrm{v}_{\beta \mathrm{i}}-\mathrm{c}_{\alpha \beta \mathrm{i}}^{2}\right),
$$

$$
\begin{gathered}
\mathrm{v}_{\alpha \mathrm{i}}=\operatorname{Var}\left(\hat{\alpha}_{\mathrm{i}, \text { ols }}\right)=\sigma_{1}^{2}+\sigma^{2}\left[1 / \mathrm{n}_{\mathrm{i}}+\overline{\mathrm{t}}_{\mathrm{i}}^{2} /\left\{\left(\mathrm{n}_{\mathrm{i}}-1\right) \mathrm{s}_{\mathrm{ti}}^{2}\right\}\right], \\
\mathrm{v}_{\beta \mathrm{i}}=\operatorname{Var}\left(\hat{\beta}_{\mathrm{i}, \text { ols }}\right)=\sigma_{2}^{2}+\sigma^{2} /\left\{\left(\mathrm{n}_{\mathrm{i}}-1\right) \mathrm{s}_{\mathrm{ti}}^{2}\right\}, \\
\mathrm{c}_{\alpha \beta \mathrm{i}}=\operatorname{Cov}\left(\hat{\alpha}_{\mathrm{i}, \text { ols }}, \hat{\beta}_{\mathrm{i}, \text { ols }}\right)=\sigma_{12}-\overline{\mathrm{t}}_{\mathrm{i}} \sigma^{2} /\left\{\left(\mathrm{n}_{\mathrm{i}}-1\right) \mathrm{s}_{\mathrm{ti}}^{2}\right\},
\end{gathered}
$$

and where $\bar{t}_{\mathrm{i}}$ and $\mathrm{s}_{\mathrm{ti}}^{2}$ denote the sample mean and variance of the observation times $\mathbf{t}_{\mathrm{i}}=\left(\mathrm{t}_{\mathrm{i} 1}, \ldots, \mathrm{t}_{\mathrm{in}_{\mathrm{i}}}\right)^{\prime}$. Similarly, it can be shown that

$$
\tilde{\alpha}_{i}=\mathrm{E}\left(\alpha_{\mathrm{i}} \mid \mathbf{Y}_{\mathrm{i}}\right)=\tau_{\mathrm{i} 1}+\tau_{\mathrm{i} 2} \hat{\alpha}_{\mathrm{i}, \mathrm{ols}}+\tau_{\mathrm{i} 3} \hat{\beta}_{\mathrm{i}, \mathrm{ols}}
$$

with

$$
\begin{aligned}
& \tau_{\mathrm{i} 2}=\left(\sigma_{1}^{2} \mathrm{v}_{\beta \mathrm{i}}-\sigma_{12} \mathrm{c}_{\alpha \beta \mathrm{i}}\right) / \delta_{\mathrm{i}}, \\
& \tau_{\mathrm{i} 3}=\left(\sigma_{12} \mathrm{v}_{\alpha \mathrm{i}}-\sigma_{1}^{2} \mathrm{c}_{\alpha \beta \mathrm{i}}\right) / \delta_{\mathrm{i}},
\end{aligned}
$$

and

$$
\tau_{\mathrm{i} 1}=\alpha\left(1-\tau_{\mathrm{i} 2}\right)-\beta \tau_{\mathrm{i} 3} .
$$

Consider the problem of predicting the unknown response under model (3) for subject $i$ at some clinically or otherwise significant point in time $\left(t_{i}^{*}\right)$. In other words, seeking to predict the value of

$$
\mathrm{Y}_{\mathrm{it}}^{*}=\mathrm{E}\left(\mathrm{Y}_{\mathrm{ij}} \mid \alpha_{\mathrm{i}}, \beta_{\mathrm{i}}, \mathrm{t}_{\mathrm{ij}}=\mathrm{t}_{\mathrm{i}}^{*}\right)=\alpha_{\mathrm{i}}+\beta_{\mathrm{i}} \mathrm{t}_{\mathrm{i}}^{*} .
$$

The posterior mean of $\mathrm{Y}_{\mathrm{it}}^{*}$ is

$$
\tilde{Y}_{i t}^{*}=E\left(Y_{i t}^{*} \mid Y_{i}\right)=\widetilde{\alpha}_{i}+\widetilde{\beta}_{i} t_{i}^{*}
$$

where $\widetilde{\beta}_{i}$ and $\widetilde{\alpha}_{i}$ are as defined in (5) and (6), for $n_{i} \geq 2$. EBLUPs for $\widetilde{\beta}_{i}$ and $\widetilde{\alpha}_{i}$ are obtained by inserting parameter estimates into the general expressions for $E\left(\beta_{i} \mid Y_{i}\right)$ and $E\left(\alpha_{i} \mid Y_{i}\right)$, where $\mathrm{n}_{\mathrm{i}}=1$ is permissible. The EBLUP for $\mathrm{Y}_{\mathrm{it}}^{*}$ inserts the EBLUPs for $\widetilde{\beta}_{i}$ and $\widetilde{\alpha}_{i}$ into (7).

Constrained Bayes Predictions

The constrained Bayes (CB) approach (Louis, 1984) was extended by Ghosh (1992) 


\section{BAYES PREDICTIONS OF RANDOM INTERCEPTS AND SLOPES}

into a flexible paradigm. Lyles and Xu (1999) suggested that this general idea provides a natural alternative to the EBLUP in the mixed linear models context when overshrinkage could detract from the desired application of predicted values. They applied a slight adaptation of the CB concept under models (1) and (3) by minimizing prediction mean squared error (MSEP) among unbiased candidates whose variances match that of the assumed random effects distribution. While this necessarily results in some sacrifice in overall MSEP relative to the posterior mean, it provides a set of predictions that more faithfully reproduce the underlying distribution of interest and are less likely to under-represent the extremeness of experimental units in the tails.

Under model (1), the CB predictor for $\mu_{\mathrm{i}}$ recommended by Lyles and $\mathrm{Xu}$ is obtained directly by forcing the first two moments of the $\tilde{\mu}_{\mathrm{i}}$ and $\mu_{\mathrm{i}}$ distributions to match:

$$
\tilde{\mu}_{i, L X}=\sqrt{v_{i}} \bar{y}_{i}+\left(1-\sqrt{v_{i}}\right) \mu
$$

The square root is indicative of the reduction in shrinkage relative to the posterior mean in (2). Under model (3), use of a Lagrangian multiplier to enforce equality of the second moments while minimizing MSEP yields a constrained Bayes alternative to the posterior mean in (5):

$$
\widetilde{\beta}_{\mathrm{i}, \mathrm{LX}}=\gamma_{\mathrm{i} 1}+\gamma_{\mathrm{i} 2} \hat{\alpha}_{\mathrm{i}, \mathrm{ols}}+\gamma_{\mathrm{i} 3} \hat{\beta}_{\mathrm{i}, \mathrm{ols}}
$$

The coefficients in (9) are defined as

$$
\begin{gathered}
\gamma_{i 1}=\beta\left(1-\gamma_{\mathrm{i} 3}\right)-\alpha \gamma_{\mathrm{i} 2}, \\
\gamma_{\mathrm{i} 2}= \pm \eta_{\mathrm{i}}\left[\sigma_{2}^{2} /\left\{\mathrm{v}_{\beta \mathrm{i}}+\eta_{\mathrm{i}}\left(2 \mathrm{c}_{\alpha \beta \mathrm{i}}+\eta_{\mathrm{i}} \mathrm{v}_{\alpha \mathrm{i}}\right)\right\}\right]^{1 / 2}
\end{gathered}
$$

and

where

$$
\gamma_{\mathrm{i} 3}=\gamma_{\mathrm{i} 2} / \eta_{\mathrm{i}}
$$

$$
\eta_{\mathrm{i}}=\left(\mathrm{v}_{\beta \mathrm{i}} \sigma_{12}-\sigma_{2}^{2} \mathrm{c}_{\alpha \beta \mathrm{i}}\right)\left(\mathrm{v}_{\alpha \mathrm{i}} \sigma_{2}^{2}-\sigma_{12} \mathrm{c}_{\alpha \beta \mathrm{i}}\right)^{-1} .
$$

The \pm sign in front of $\gamma_{\mathrm{i} 2}$ is needed because there are two roots, although the positive root is usually correct. The positive or negative root is taken for $\gamma_{\mathrm{i} 2}$ depending on which yields the lower value of the MSEP criterion:

$$
\begin{aligned}
& \operatorname{MSEP}=\mathrm{E}\left(\tilde{\beta}_{\mathrm{i}}-\beta_{\mathrm{i}}\right)^{2} \\
& =\left(\gamma_{\mathrm{i} 2}^{2} \mathrm{v}_{\alpha \mathrm{i}}+\gamma_{\mathrm{i} 3}^{2} \mathrm{v}_{\beta \mathrm{i}}+2 \gamma_{\mathrm{i} 2} \gamma_{\mathrm{i} 3} \mathrm{c}_{\alpha \beta \mathrm{i}}\right)-2\left(\gamma_{\mathrm{i} 2} \sigma_{12}+\gamma_{\mathrm{i} 3} \sigma_{2}^{2}\right)+\sigma_{2}^{2}
\end{aligned}
$$

The definitions of $\eta_{i}$ and $\gamma_{\mathrm{i} 2}$ serve to correct a subtle error in the result originally put forth by Lyles and Xu (1999). The Appendix provides analogous constrained Bayes predictors for $\alpha_{i}$ and $Y_{i t}^{*}$, which are both new to the literature. Empirical constrained Bayes (ECB) predictions are obtained for practical use by replacing unknown parameters by their estimates in equations (8), (9), (A1), and (A3), and when calculating the MSEP criterion in (10).

In contrast to the preceding direct model-specific CB predictors, consider the general CB paradigm provided by Ghosh (1992). Using $\beta_{\mathrm{i}}$ under model (3) to illustrate, $\widetilde{\beta}_{\mathrm{i}, \mathrm{B}}$ is first taken to indicate the posterior mean (or Bayes) predictor for subject i. An algebraic expression for $\widetilde{\beta}_{\mathrm{i}, \mathrm{B}}$ was given in (5). Ghosh's approach defines the $\mathrm{CB}$ estimate $\left(\widetilde{\beta}_{\mathrm{i}, \mathrm{G}}\right)$ as follows:

$$
\widetilde{\beta}_{i, G}=w \widetilde{\beta}_{i, B}+(1-w) \overline{\widetilde{\beta}}_{B}
$$

where

$$
\begin{gathered}
\overline{\widetilde{\beta}}_{\mathrm{B}}=\mathrm{k}^{-1} \sum_{\mathrm{h}=1}^{\mathrm{k}} \widetilde{\beta}_{\mathrm{h}, \mathrm{B}}, \mathrm{w}=\left(1+\mathrm{H}_{1} / \mathrm{H}_{2}\right)^{1 / 2}, \\
\mathrm{H}_{2}=\sum_{\mathrm{h}=1}^{\mathrm{k}}\left(\widetilde{\beta}_{\mathrm{h}, \mathrm{B}}-\overline{\widetilde{\beta}}_{\mathrm{B}}\right)^{2},
\end{gathered}
$$

and

$$
\mathrm{H}_{1}=\operatorname{tr}\left\{\operatorname{Var}\left(\boldsymbol{\beta}-\bar{\beta} \mathbf{1}_{\mathrm{k}} \mid \mathbf{Y}\right)\right\}=\left(1-\mathrm{k}^{-1}\right) \sum_{\mathrm{h}=1}^{\mathrm{k}} \operatorname{Var}\left(\beta_{\mathrm{h}} \mid \mathbf{Y}_{\mathrm{h}}\right)
$$

with $\quad \boldsymbol{\beta}$ representing the k-vector $\left(\beta_{1}, \beta_{2}, \ldots, \beta_{\mathrm{k}}\right)^{\prime}$. 


\section{LYLES, MOORE, MANATUNGA \& EASLEY}

The latter equality is supplied in (12) as a result of assumed independence across experimental units for the class of mixed models under consideration here. Note that in addition to the posterior means, this paradigm requires only the corresponding posterior variances. Using the previous notation (see equation (4) and Appendix), results in:

$$
\begin{aligned}
& \operatorname{Var}\left(\beta_{\mathrm{i}} \mid \mathbf{Y}_{\mathrm{i}}\right)= \\
& \sigma_{2}^{2}-\left\{\sigma_{12} \mathbf{1}_{\mathrm{n}_{\mathrm{i}}}^{\prime}+\sigma_{2}^{2} \mathbf{t}_{\mathrm{i}}^{\prime}\right\} \boldsymbol{\Sigma}_{\mathrm{i}}^{-1}\left\{\sigma_{12} \mathbf{1}_{\mathrm{n}_{\mathrm{i}}}^{\prime}+\sigma_{2}^{2} \mathbf{t}_{\mathrm{i}}^{\prime}\right\}^{\prime}, \\
& \operatorname{Var}\left(\alpha_{\mathrm{i}} \mid \mathbf{Y}_{\mathrm{i}}\right)= \\
& \sigma_{1}^{2}-\left\{\sigma_{1}^{2} \mathbf{1}_{\mathrm{n}_{\mathrm{i}}}^{\prime}+\sigma_{12} \mathbf{t}_{\mathrm{i}}^{\prime}\right\} \boldsymbol{\Sigma}_{\mathrm{i}}^{-1}\left\{\sigma_{1}^{2} \mathbf{1}_{\mathrm{n}_{\mathrm{i}}}^{\prime}+\sigma_{12} \mathbf{t}_{\mathrm{i}}^{\prime}\right\}^{\prime},
\end{aligned}
$$

and

$$
\begin{aligned}
& \operatorname{Var}\left(\mathrm{Y}_{\mathrm{it}}^{*} \mid \mathbf{Y}_{\mathrm{i}}\right)= \\
& \operatorname{Var}\left(\mathrm{Y}_{\mathrm{it}}^{*}\right)-\left\{\psi_{\mathrm{i} 1} \mathbf{1}_{\mathrm{n}_{\mathrm{i}}}^{\prime}+\psi_{\mathrm{i} 2} \mathbf{t}_{\mathrm{i}}^{\prime}\right\} \boldsymbol{\Sigma}_{\mathrm{i}}^{-1}\left\{\psi_{\mathrm{i} 1} \mathbf{1}_{\mathrm{n}_{\mathrm{i}}}^{\prime}+\psi_{\mathrm{i} 2} \mathbf{t}_{\mathrm{i}}^{\prime}\right\}^{\prime} .
\end{aligned}
$$

ECB predictions for practical use can be obtained by replacing unknown parameters by their estimates when computing the posterior means and variances, and the building blocks for these calculations are already built into standard software for mixed linear models.

Incorporating Fixed or Time-Dependent Covariates

Consider the following extensions of models (1) and (3) to include a set of $\mathrm{T}$ covariates, some of which may be timedependent:

$$
\begin{aligned}
& Y_{i j}=\mu+b_{i}+\sum_{t=1}^{T} \theta_{t} c_{i j t}+e_{i j} \\
& Y_{i j}=\left(\alpha+a_{i}\right)+\left(\beta+b_{i}\right) t_{i j}+\sum_{t=1}^{T} \theta_{t} c_{i j t}+e_{i j}
\end{aligned}
$$

where $c_{i j t}$ represents the observed value of the $t^{\text {th }}$ covariate for subject $\mathrm{i}$ at time point $\mathrm{j}(\mathrm{t}=1, . ., \mathrm{T}$; $\left.\mathrm{i}=1, . ., \mathrm{k} ; \mathrm{j}=1, . ., n_{\mathrm{i}}\right)$. Let $\mathbf{c}_{\mathrm{ij}}{ }^{\prime}=\left(\mathrm{c}_{\mathrm{ij} 1}, \mathrm{c}_{\mathrm{ij} 2}, \ldots, \mathrm{c}_{\mathrm{ijT}}\right)$ and form the $\mathrm{n}_{\mathrm{i}} \times \mathrm{T}$ matrix $\mathbf{C}_{\mathrm{i}}$ by stacking the row vectors $\mathbf{c}_{\mathrm{ij}}$ in order. Next, define the transformed observed data vector $\mathbf{y}_{\mathrm{i}}^{\bullet}=\mathbf{y}_{\mathrm{i}}-\mathbf{C}_{\mathrm{i}} \boldsymbol{\theta}, \quad$ where $\boldsymbol{\theta}=\left(\theta_{1}, \theta_{2}, \ldots, \theta_{\mathrm{T}}\right)^{\prime}$. The extension to the posterior mean formula in (2) is

$$
\tilde{\mu}_{\mathrm{i}}=\mathrm{E}\left(\mu_{\mathrm{i}} \mid \mathbf{Y}_{\mathrm{i}}, \mathbf{C}_{\mathrm{i}}\right)=v_{\mathrm{i}} \overline{\mathrm{y}}_{\mathrm{i}}^{\bullet}+\left(1-v_{\mathrm{i}}\right) \mu
$$

with $\mu_{\mathrm{i}}$ and $v_{\mathrm{i}}$ defined exactly as before and $\overline{\mathrm{y}}_{\mathrm{i}}^{\bullet}=\mathrm{n}_{\mathrm{i}}^{-1} \sum_{\mathrm{j}=1}^{\mathrm{n}_{\mathrm{i}}} \mathrm{y}_{\mathrm{ij}}^{\bullet}$. In practice, predicting $\widetilde{Y}_{i j}=E\left(Y_{i j} \mid b_{i}, \mathbf{C}_{i}\right)=\mu_{i}+\mathbf{c}_{i j}{ }^{\prime} \boldsymbol{\theta}$ may be more likely. Standard mixed linear model software typically provides the EBLUP for $b_{i}$, from which EBLUPs for $\mu_{\mathrm{i}}$ and $\widetilde{\mathrm{Y}}_{\mathrm{ij}}$ are easily obtained.

Similarly, extensions to (4) and (5) under the randomized regression model (17) are

$$
\begin{aligned}
\tilde{\beta}_{\mathrm{i}} & =\mathrm{E}\left(\beta_{\mathrm{i}} \mid \mathbf{Y}_{\mathrm{i}}, \mathbf{C}_{\mathrm{i}}\right) \\
& =\beta+\left(\sigma_{12} \mathbf{1}_{\mathrm{n}_{\mathrm{i}}}^{\prime}+\sigma_{2}^{2} \mathbf{t}_{\mathrm{i}}^{\prime}\right) \boldsymbol{\Sigma}_{\mathrm{i}}^{-1}\left(\mathbf{y}_{\mathrm{i}}^{\bullet}-\alpha \mathbf{1}_{\mathrm{n}_{\mathrm{i}}}-\beta \mathbf{t}_{\mathrm{i}}\right)
\end{aligned}
$$

and

$$
\begin{aligned}
\tilde{\beta}_{i} & =E\left(\beta_{i} \mid \mathbf{Y}_{i}, \mathbf{C}_{i}\right) \\
& =\gamma_{i 1}+\gamma_{i 2} \hat{\alpha}_{i, 0 l s}+\gamma_{i 3} \hat{\beta}_{i, \text { ols }}
\end{aligned}
$$

where $\beta_{i}, \gamma_{i 1}, \gamma_{i 2}$, and $\gamma_{i 3}$ are defined as before, but with $\hat{\alpha}_{\mathrm{i} \text { ols }}$ and $\hat{\beta}_{\mathrm{i} \text {,ols }}$ now representing the OLS intercept and slope from regressing $\mathbf{y}_{\mathbf{i}}^{\bullet}$ on $\mathbf{t}_{\mathrm{i}}$. The algebraic expression in (19) requires $\mathrm{n}_{\mathrm{i}} \geq$ 2. Standard software typically provides EBLUPs for $a_{i}$ and $b_{i}$, from which EBLUPs for $\alpha_{i}$ and $\beta_{i}$ follow directly. In turn, the analogue to equation (7) becomes

$$
\begin{aligned}
\tilde{Y}_{\mathrm{it}}^{*} & =\mathrm{E}\left(\mathrm{Y}_{\mathrm{it}}^{*} \mid \mathbf{Y}_{\mathrm{i}}, \mathbf{C}_{\mathrm{i}}\right) \\
& =\tilde{\alpha}_{\mathrm{i}}+\tilde{\beta}_{\mathrm{i}} \mathrm{t}_{\mathrm{i}}^{*}+\mathbf{c}_{\mathrm{i}, \mathrm{t}} \boldsymbol{\theta}
\end{aligned}
$$

which can arguably be defined only for nontime-dependent covariates unless the values of 


\section{BAYES PREDICTIONS OF RANDOM INTERCEPTS AND SLOPES}

any time dependent ones are known at time $t_{i}^{*}$ (as indicated by the notation $\mathbf{c}_{\mathrm{i}, \mathrm{t}} *$ ).

Extensions of the $\mathrm{CB}$ predictors $\tilde{\mu}_{i, L X}, \widetilde{\beta}_{i, L X}$, and $\widetilde{\alpha}_{i, L X}$ in equations (8), (9), and (A1) with covariate adjustment according to models (16) and (17) require no changes to the coefficients already given, once the transformation $\mathbf{y}_{\mathrm{i}}^{\bullet}=\mathbf{y}_{\mathrm{i}}-\mathbf{C}_{\mathrm{i}} \boldsymbol{\theta}$ is made. The same is true for $\tilde{Y}_{i t, L X}^{*}$ in equation (A3), except the term $\mathbf{c}_{\mathrm{i}, \mathrm{t}} * \boldsymbol{\theta}$ is added as in (20). ECB predictions for practical use follow, once estimates of the mixed linear model parameters are inserted.

In adapting the paradigm of Ghosh (1992) as in (11) and (12), ECB predictions appear straightforward for a broad class of general linear mixed models because (i) EBLUPs accounting for covariates come directly out of standard software, and (ii) the required conditional variances [e.g., (13)-(15)] are unchanged by the addition of covariates. In the case of $\widetilde{Y}_{i t}^{*}$, Ghosh's paradigm requires a separate application of posterior mean and variance calculations analogous to those in (11) and (12) for each unique value of $t_{i}^{*}$ (Moore, 2006).

\section{Example}

Consider longitudinal data on CD4 cell counts collected for the Pediatric Pulmonary and Cardiovascular Complications of Vertically Transmitted $\left(\mathrm{P}^{2} \mathrm{C}^{2}\right)$ HIV Infection Study (The $\mathrm{P}^{2} \mathrm{C}^{2}$ Study Group, 1996). This National Heart, Lung, and Blood Institute-funded study enrolled infants born to HIV-positive women during the years 1990-1993, and followed them prospectively during the first few years of life. Specifically, data was analyzed on 59 vertically infected infants who contributed a total of 539 CD4 counts over time, with the number of measurements per child ranging from 3 to 19 . Initial CD4 counts were typically observed at or within a few weeks of birth. The length of follow-up on children ranged from 1 to 6 years, with a median of 3.5 years. Also recorded for each child was the age at which he or she was determined to have reached Class A (mildly symptomatic) HIV status (Centers for Disease Control and Prevention, 1994). Across the 59 subjects, this age ranged from 0.4 to 16 months.

A mixed linear model was fit to these data, with age as the longitudinal metameter. While there was some indication of right skewness in the CD4 counts, standard transformations tended to overcorrect this and for the sake of clarity the untransformed CD4 counts were analyzed. For an illustration with covariate adjustment, the child's gender ( 1 for male, 0 for female) and the concurrent CD8 cell count were accounted for via the following model:

$$
\begin{aligned}
& \mathrm{CD}_{\mathrm{ij}}= \\
& \left(\alpha+\mathrm{a}_{\mathrm{i}}\right)+\left(\beta+\mathrm{b}_{\mathrm{i}}\right) \mathrm{AGE}_{\mathrm{ij}}+\theta_{1} \operatorname{GENDER}_{\mathrm{i}}+\theta_{2} \mathrm{CD} 8_{\mathrm{ij}}+\mathrm{e}_{\mathrm{ij}}
\end{aligned}
$$

The primary objective was to compare EBLUP and ECB predictions of the random intercepts $\left(\alpha_{i}=\alpha+a_{i}\right)$ and random slopes $\left(\beta_{i}=\beta+b_{i}\right)$. For this purpose, both the direct ECB approach patterned after Lyles and Xu (1999; 'LX ECB') and the general ECB method following Ghosh (1992) were investigated.

Next, EBLUP and Ghosh ECB predictions of $\mathrm{Y}_{\mathrm{it}}^{*}$ were compared, where $\mathrm{Y}_{\mathrm{it}}^{*}=$ $\alpha_{\mathrm{i}}+\beta_{\mathrm{i}} \mathrm{t}_{\mathrm{i}}^{*}+\theta_{1} \mathrm{GENDER}_{\mathrm{i}}+\theta_{2} \mathrm{CD} 8_{\mathrm{i}}$ represents the unknown model-based CD4 count at time $\mathrm{t}_{\mathrm{i}}^{*}$. For this latter purpose, $\mathrm{t}_{\mathrm{i}}^{*}$ was defined as the age at which the child was diagnosed with Class A HIV disease, and model (21) was re-fit with the initial $\mathrm{CD} 8$ count $\left(\mathrm{CD} 8_{\mathrm{i}}\right)$ in place of the time-dependent version in light of the fact that CD8 was unrecorded at the times $t_{i}^{*}$. Table 1 provides the coefficient and variance component estimates from fitting both versions of model (21) by maximum likelihood via SAS PROC MIXED (SAS Institute, Inc., 2004a). The table indicates a highly significant average decline of approximately 400 CD4 cells per year, little effect of gender, and a significant positive association with the CD8 count, regardless of 


\section{LYLES, MOORE, MANATUNGA \& EASLEY}

whether the latter was measured only initially or treated as time-dependent.

In Figure 1A, EBLUPs are plotted for the random intercepts $\alpha_{i}$ against the corresponding Ghosh ECB predictions, based on the model treating CD8 as time-dependent. The EBLUPs were obtained directly from the mixed linear model software, and the Ghosh ECBs were computed readily using the EBLUPs and posterior variance calculations with variance components replaced by their MLEs (see e.g., eqns. 11-15). The reduction in shrinkage afforded by the CB method is evidenced by the characteristic tilting in the pattern of plotted points.

Figure 1B plots the LX ECB predictions of $\alpha_{i}$ versus the Ghosh ECBs. To obtain the LX ECBs, the MLEs for variance components were inserted into the formulae provided herein, with covariate adjustment as described in Section 2.3. With a few exceptions, the two approaches produce essentially identical results. The sample means of the 59 EBLUP, Ghosh ECB, and LX ECB predicted values were $1675.5,1675.5$, and 1675.3, respectively. The corresponding sample variances were 365470,475026 , and 473752 . Comparing these to $\hat{\alpha}=1675.5$ and $\hat{\sigma}_{1}^{2}=468832$ (Table 1) highlights the moment matching characteristics of the $\mathrm{CB}$ approaches, as well as the overshrinkage of the EBLUP.

Figure 2 is the counterpart to Figure 1, for the predicted random slopes $\left(\beta_{\mathrm{i}}\right)$. The tilting remains prominent in Figure 2A, while Figure $2 \mathrm{~B}$ reveals somewhat more pronounced discrepancies between the Ghosh and LX ECB point predictions than in the case of the intercepts. The sample means of the EBLUP, Ghosh ECB, and LX ECB predicted values were $-388.2,-388.2$, and -395.3 , respectively, with sample variances of 27904, 48316, and 49401. Comparing these to $\hat{\beta}=-388.2$ and $\hat{\sigma}_{2}^{2}=47843$ (Table 1) again highlights the ECB moment-matching properties in action.

Figure 3 illustrates the reduction in shrinkage of the Ghosh ECB predictions (open circles) of CD4 cell counts at the time of Class A disease $\left(Y_{i t}^{*}\right)$, relative to the EBLUPs (closed circles). Separate plots are presented for females and males, with overlays of the population average regression lines calculated at the overall mean of the 59 initial CD8 counts (1294.7 cells). The lines provide a relevant visual reference based on the fit of model (21) (Table 1), although the plotted points were not expected to directly follow these linear trends given that subjects with less rapidly declining CD4 counts theoretically reach Class A disease at later ages.

\section{Results}

While the close agreement of the sample means and variances of the ECB predictions to the corresponding estimated moments $\left(\hat{\alpha}\right.$ and $\hat{\sigma}_{1}^{2}$, $\hat{\beta}$ and $\hat{\sigma}_{2}^{2}$ ) in the real-data example is indicative, simulation studies are required to further assess the quality of the variance match and to compare the performances of the Ghosh and LX ECB methods in practical settings. Several combinations of covariates and true parameter values were examined and qualitatively similar results were found. In the interest of brevity and relevance to the application presented in the previous section, simulations designed to mimic the conditions observed in the example are summarized. Simulations were carried out using matrix manipulations and standard random number generating functions available in the SAS IML package (SAS Institute, Inc., 2004b).

Performance comparison: LX vs. Ghosh CB predictors

Data was generated according to model (21) for 20,000 hypothetical subjects, with true parameter values equal to the estimates listed in the top half of Table 1. The fabricated CD4 data were unbalanced with $\mathrm{n}_{\mathrm{i}}$ ranging randomly between 2 and 10, and measurements were unequally timed over approximate 2 month intervals. Simulated subjects were male or female with probability 0.5 . For simplicity, timevarying CD8 counts were generated at each visit from a normal distribution mimicking the sample mean and variance of the initial CD8 counts in the actual example. To illustrate results for predicting $\mathrm{Y}_{\mathrm{it}}^{*}$, the same simulation exercise was repeated except with a time independent 
BAYES PREDICTIONS OF RANDOM INTERCEPTS AND SLOPES

Table 1: Summary of mixed linear models fit to CD4 cell count data *

\begin{tabular}{|c|cccc|}
\hline \multirow{2}{*}{ Model $^{\dagger}$} & Coefficient & $\begin{array}{c}\text { Estimate } \\
\text { (standard error) }\end{array}$ & $\begin{array}{c}\text { Variance } \\
\text { Component }\end{array}$ & Estimate \\
\hline \multirow{4}{*}{$\begin{array}{c}\text { CD8 as time- } \\
\text { dependent }\end{array}$} & $\alpha$ & $\begin{array}{c}1675.50 \\
(138.27)\end{array}$ & $\sigma_{1}^{2}$ & 468832 \\
\cline { 2 - 5 } & $\beta$ & $\begin{array}{c}-388.17 \\
(38.06)\end{array}$ & $\sigma_{2}^{2}$ & 47843 \\
\cline { 2 - 5 } & $\theta_{1}$ & $\begin{array}{c}-163.41 \\
(146.61)\end{array}$ & $\sigma_{12}$ & -103226 \\
\hline \multirow{4}{*}{$\begin{array}{c}\text { CD8 as time- } \\
\text { independent } \\
\text { (initial value) }\end{array}$} & $\theta_{2}$ & $\begin{array}{c}0.26 \\
(0.03)\end{array}$ & $\sigma^{2}$ & 477810 \\
\cline { 2 - 5 } & $\alpha$ & $\begin{array}{c}1735.88 \\
(188.60)\end{array}$ & $\sigma_{1}^{2}$ & 429957 \\
\cline { 2 - 5 } & $\theta_{1}$ & $\begin{array}{c}-417.51 \\
(40.57)\end{array}$ & $\sigma_{2}^{2}$ & 55206 \\
\cline { 2 - 5 } & $\theta_{2}$ & $\begin{array}{c}-105.28 \\
(146.61)\end{array}$ & $\sigma_{12}$ & -102537 \\
\hline
\end{tabular}

* Data from $\mathrm{P}^{2} \mathrm{C}^{2}$ HIV Infection Study (The $\mathrm{P}^{2} \mathrm{C}^{2}$ Study Group, 1996)

$\uparrow \mathrm{CD}_{\mathrm{ij}}=\left(\alpha+\mathrm{a}_{\mathrm{i}}\right)+\left(\beta+\mathrm{b}_{\mathrm{i}}\right) \mathrm{AGE}_{\mathrm{ij}}+\theta_{1} \mathrm{GENDER}_{\mathrm{i}}+\theta_{2} \mathrm{CD} 8+\mathrm{e}_{\mathrm{ij}}$

Figure 1: EBLUP (panel A) and LX ECB (panel B) vs. Ghosh ECB predictions for random intercepts $\left(\alpha_{i}\right)$ based on the fit of model $(21)$ with CD8 count as time-dependent

EBLUP vs. Ghosh ECB

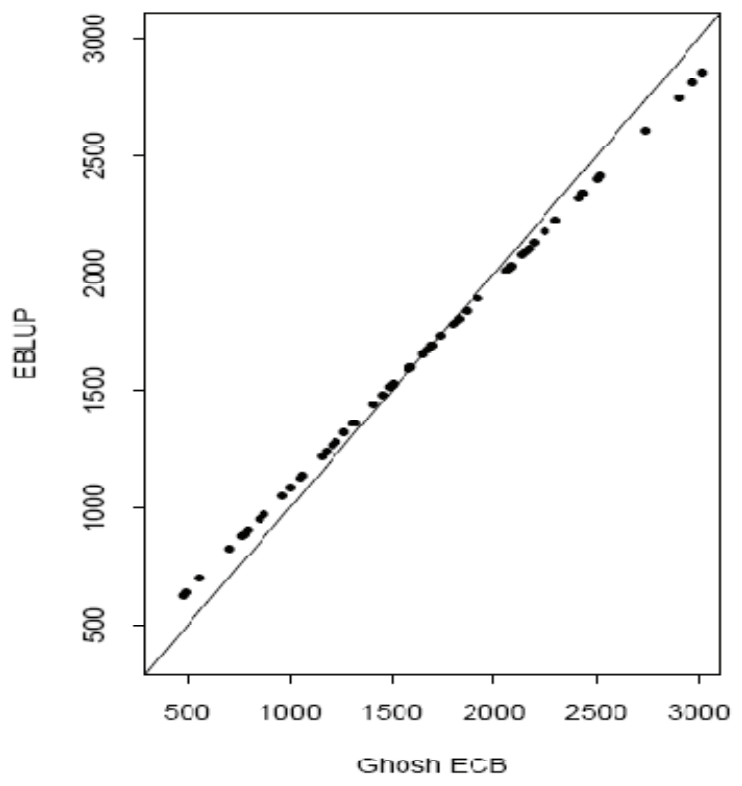

LX vs. Ghosh ECB

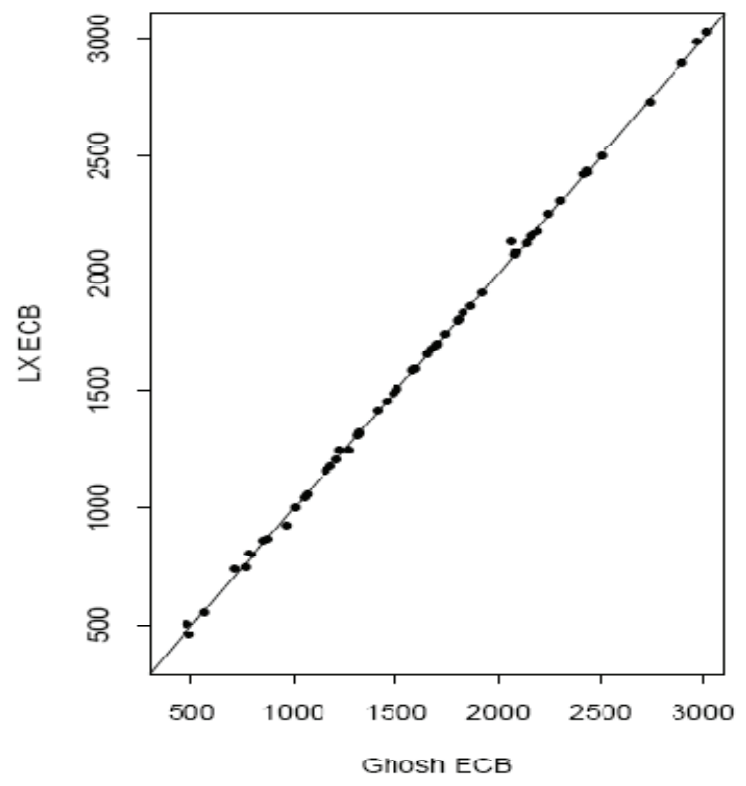




\section{LYLES, MOORE, MANATUNGA \& EASLEY}

Figure 2: EBLUP (panel A) and LX ECB (panel B) vs. Ghosh ECB Predictions for Random Slopes $\left(\beta_{\mathrm{i}}\right)$ Based on the Fit of Model (21) with CD8 Count as Time-Dependent

EBLUP vs. Ghosh ECB

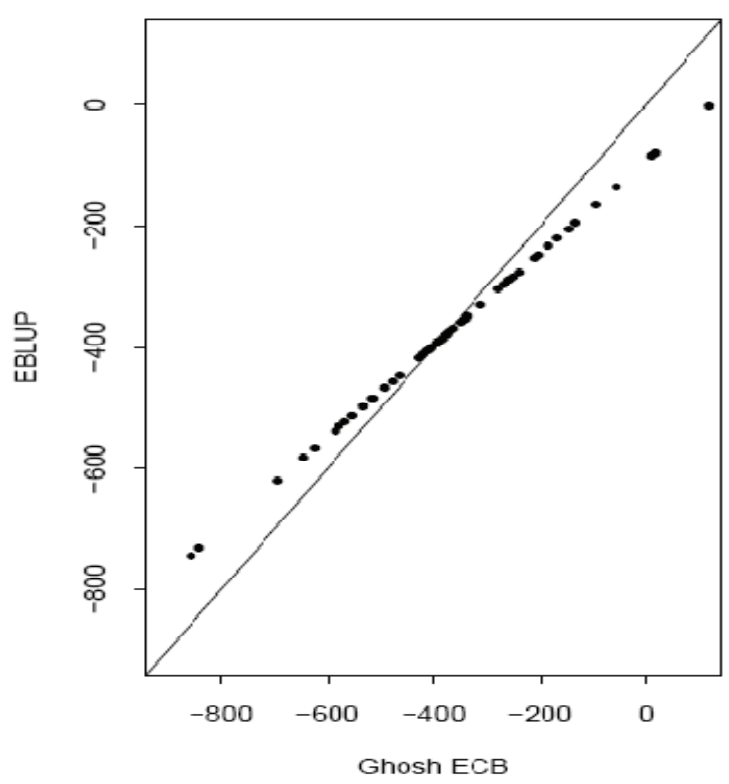

LX vs. Ghosh ECB

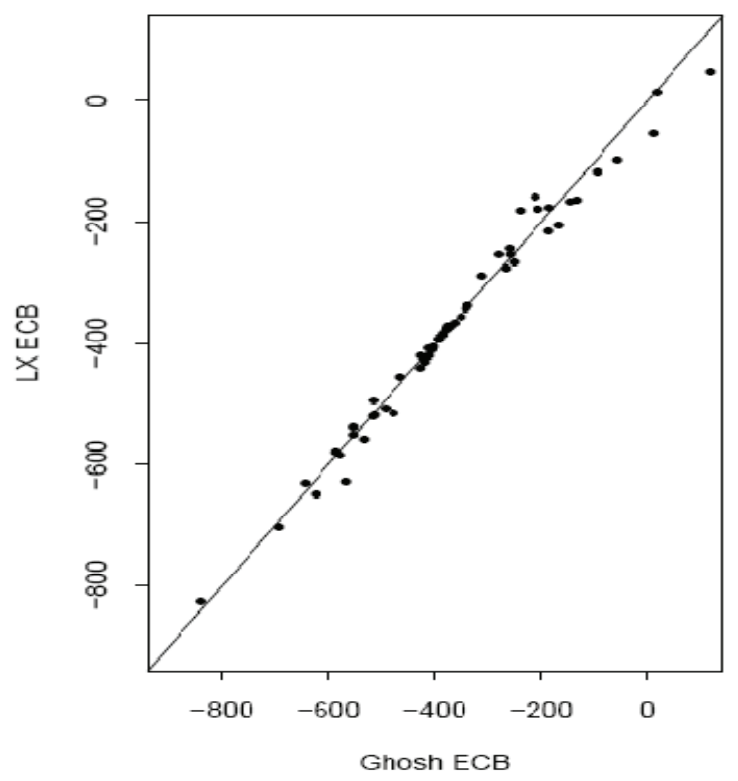

Figure 3: EBLUP (dark circle) vs. Ghosh ECB (open circle) Predictions of

$\mathrm{Y}_{\mathrm{it}}^{*}=\alpha_{\mathrm{i}}+\beta_{\mathrm{i}} \mathrm{t}_{\mathrm{i}}^{*}+\theta_{1} \mathrm{GENDER}_{\mathrm{i}}+\theta_{2} \mathrm{CD} 8_{\mathrm{i}}$ for Females (panel A) and Males (panel B), with Initial CD8 Count as a Time-Independent Covariate

EBLUP vs. Ghosh ECB for Females

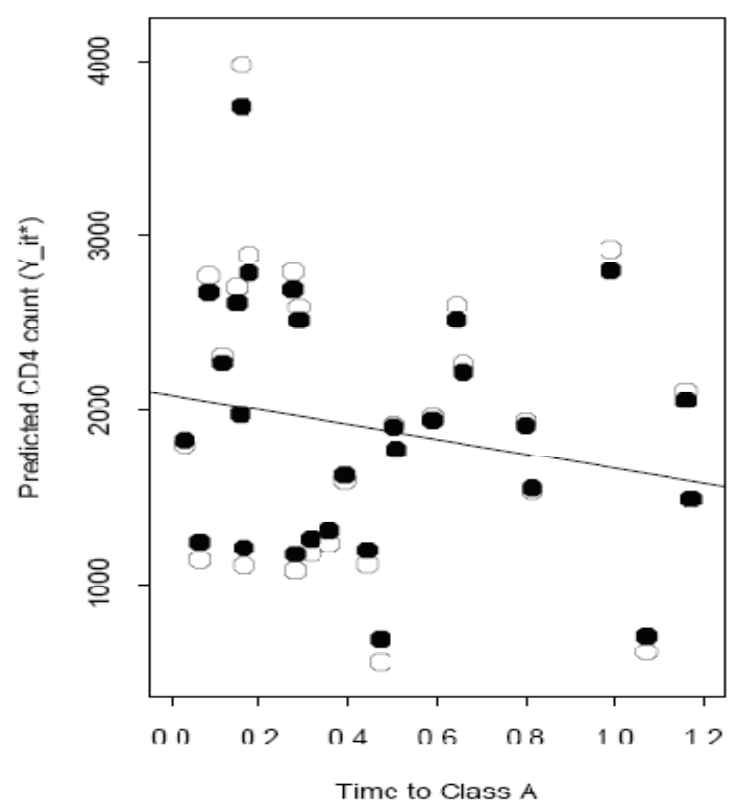

EBLUP vs. Ghosh ECB for Males

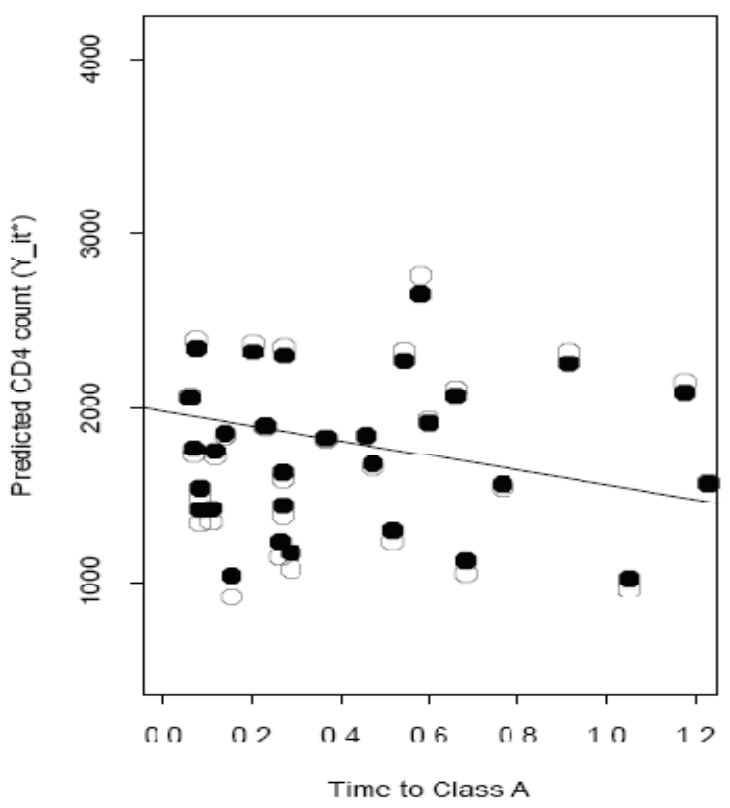




\section{BAYES PREDICTIONS OF RANDOM INTERCEPTS AND SLOPES}

initial CD8 count in place of the time-varying version. The time point of interest $\left(t_{i}^{*}\right)$ was taken to occur at 2 years for each simulated subject.

Table 2 summarizes the simulation results for predicting the $\alpha_{i}$ 's and $\beta_{i}$ 's, and Table 3 summarizes the results for predicting $\mathrm{Y}_{\mathrm{it}}^{*}$. In each case, the sample means of the BLUPs and the two CB predictors closely match the true mean of the random variable being predicted.
The sample variances over 20,000 simulated subjects for both the LX and Ghosh CB methods are very close to the corresponding true variances in each case, while the overshrinkage of the BLUPs is evident by their notably tighter sampling distributions. As a final note, the empirical prediction MSEs of the LX and Ghosh methods are similar, though predictably somewhat larger than those for the corresponding BLUPs. In each case, the Ghosh method achieved a small MSE advantage relative to the LX approach.

Table 2: Simulation Results for Random Intercept and Slope Predictions ${ }^{* \dagger}$

\begin{tabular}{|c|c|c|c|c|}
\hline & True $\alpha_{i}^{\prime} s$ & $\tilde{\alpha}_{i, B L U P}$ & $\tilde{\alpha}_{i, L X}$ & $\tilde{\alpha}_{i, G}$ \\
\hline Mean & 1675.5 & 1680.8 & 1681.2 & 1680.8 \\
\hline Variance & 468832 & 376252 & 475834 & 474736 \\
\hline Prediction MSE & -- & 98600 & 105400 & 104469 \\
\hline & True $\beta_{\mathrm{i}}{ }^{\prime} \mathrm{s}$ & $\widetilde{\beta}_{\mathrm{i}, \mathrm{BLUP}}$ & $\widetilde{\beta}_{\mathrm{i}, \mathrm{LX}}$ & $\widetilde{\beta}_{\mathrm{i}, \mathrm{G}}$ \\
\hline Mean & -388.2 & -389.4 & -386.2 & -389.4 \\
\hline Variance & 47843 & 16115 & 48693 & 48134 \\
\hline Prediction MSE & -- & 31593 & 40375 & 40125 \\
\hline
\end{tabular}

*Data simulated to mimic model (21) with parameters equal to estimates in Table 1 (top)

$\uparrow$ Predictions computed assuming parameter values that generated the data

Table 3: Simulation Results for $Y_{i t}^{*}$ Predictions ${ }^{* \dagger}$

\begin{tabular}{|ccccc|}
\hline & True $\mathrm{Y}_{\text {it }}^{*} \mathrm{~s}$ & $\tilde{\mathrm{Y}}_{\text {it,BLUP }}^{*}$ & $\tilde{\mathrm{Y}}_{\text {it,LX }}^{*}$ & $\tilde{\mathrm{Y}}_{\text {it,G }}^{*}$ \\
\hline Mean & 1156.4 & 1158.4 & 1158.3 & 1158.4 \\
\hline Variance & 289054 & 177184 & 289249 & 288880 \\
\hline Prediction MSE & -- & 110636 & 128884 & 124112 \\
\hline
\end{tabular}

*Data simulated to mimic model (21) with parameters equal to estimates in Table 1 (bottom)

$\dagger$ Predictions computed assuming parameter values that generated the data 


\section{LYLES, MOORE, MANATUNGA \& EASLEY}

Flexibility of Ghosh's Approach under More General Covariance Structures

The LX approach, while presentable in closed form for the models considered thus far, relies upon a strict form for candidate predictors and may be cumbersome or infeasible to extend to arbitrary mixed linear models. For example, consider an extension of model (17) to incorporate serially correlated random errors, e.g., via an $\operatorname{AR}(1)$ structure. Rather than $\sigma^{2} \mathbf{I}_{n_{i}}$, the covariance matrix of the $i^{\text {th }}$ vector of random errors $\left(\mathbf{e}_{i}\right)$ now takes the form

$$
\operatorname{Var}\left(\mathbf{e}_{\mathrm{i}}\right)=\sigma^{2}\left(\begin{array}{ccccc}
1 & \rho & \rho^{2} & \ldots & \rho^{\mathrm{n}_{\mathrm{i}}-1} \\
& 1 & \rho & \ldots & \rho^{\mathrm{n}_{\mathrm{i}}-2} \\
& & 1 & \rho & \cdot \\
& & & & \cdot \\
& & & & \cdot \\
& & & & \\
& & & & 1
\end{array}\right)=\sigma^{2} \mathbf{P}_{\mathrm{AR}(1)}
$$

The structured error covariance makes it less reasonable to restrict to the class of predictors that are linear combinations of $\hat{\alpha}_{i, o l s}$ and $\hat{\beta}_{i, o l s}$ [see eqn. (5)] in order to develop a CB predictor via the LX approach. Further, the MSEP becomes a much more difficult objective function to work with analytically.

Fortunately, the general paradigm of Ghosh (1992) encounters no difficulty with such an extension. In particular, the EBLUP remains available via common mixed linear model software, and the MVN theory-based posterior variance remains straightforward, with the only adjustment necessary to equations (13) and (14) being that the matrix $\boldsymbol{\Sigma}_{\mathrm{i}}=\operatorname{Var}\left(\mathbf{Y}_{\mathrm{i}}\right)=\mathbf{Z}_{\mathrm{i}} \Delta \mathbf{Z}_{\mathrm{i}}^{\prime}+\sigma^{2} \mathbf{I}_{\mathrm{n}_{\mathrm{i}}} \quad$ becomes $\boldsymbol{\Sigma}_{\mathrm{i}}=\mathbf{Z}_{\mathbf{i}} \Delta \mathbf{Z}_{\mathbf{i}}^{\prime}+\sigma^{2} \mathbf{P}_{\mathrm{AR}(1)}$.

Table 4 displays the results of an additional simulation under the $\mathrm{AR}(1)$ error model. Data were generated under model (21) using the same true parameter values as for the simulation summarized in the top half of Table I, except with an AR(1) error structure for the covariance matrix of the random errors. The value $\rho=0.30$ was assumed. There were 5,000 simulated subjects, each with $\mathrm{n}_{\mathrm{i}}=8$ observations. The model was fit via SAS PROC MIXED and the ECB versions of $\widetilde{\alpha}_{i, G}$ and $\widetilde{\beta}_{i, G}$ were computed as in (11) and (12), by incorporating the EBLUPs produced by the software together with the estimated posterior variances as in (13) and (14).

As Table 4 shows, excellent matches were achieved between the sample means and variances of the ECB predictions, and the corresponding estimated population moments $\left(\alpha, \beta, \sigma_{1}^{2}, \sigma_{2}^{2}\right)$. Figure 4 displays histograms of the ECBs, which almost perfectly match the overlaid estimated theoretical normal distributions. In contrast, histograms of the EBLUPs (not shown) are characterized by markedly narrow spread as expected, thus dramatically failing to match the underlying theoretical distribution. Potential drawbacks of this overshrinkage in certain applications have been discussed at length in the literature (e.g., Louis, 1984; Ghosh, 1992; Shen \& Louis, 1998; Stern \& Cressie, 1999). The current example further highlights the flexibility of the Ghosh paradigm as a general approach to ECB prediction under the mixed linear model.

\section{Conclusion}

Louis (1984) and Ghosh (1992) discussed the motivation and potential benefits of constrained Bayes estimation, which seeks to optimize a traditional MSE criterion subject to matching the posterior expectation of the first two moments of a parameter distribution to the corresponding true moments. In particular, the known overall MSE advantage of the traditional posterior mean approach (which underlies the BLUP in the mixed linear model setting) is sometimes worth sacrificing to obtain a set of predictions with a histogram more closely matching a true distribution of random effects. For specific discussions of contexts in which constrained Bayes and related approaches offer tangible appeal, see Shen and Louis (1998), Lyles and Xu (1999), Stern and Cressie (1999), and Lyles, et al. (2007). 
BAYES PREDICTIONS OF RANDOM INTERCEPTS AND SLOPES

Table 4: Simulation Results for Random Intercept and Slope Predictions Under AR(1) Error Model ${ }^{* \dagger}$

\begin{tabular}{|cccc|}
\hline $\begin{array}{c}\text { Parameter } \\
\text { Estimates }\end{array}$ & $\begin{array}{c}\text { ECB Sample } \\
\text { Moments }\end{array}$ & $\widetilde{\alpha}_{\mathrm{i}, \mathrm{G}}$ & $\widetilde{\beta}_{\mathrm{i}, \mathrm{G}}$ \\
\hline$\hat{\alpha}=1683.04$ & Mean & 1683.04 & -389.21 \\
$\hat{\sigma}_{1}^{2}=481899$ & Variance & 481961 & 53556 \\
\hline$\hat{\beta}=-389.21$ & & & \\
$\hat{\sigma}_{2}^{2}=53551$ & & & \\
\hline
\end{tabular}

*Data simulated to mimic model (21) with $\mathrm{k}=5000, \mathrm{n}_{\mathrm{i}}=8(\forall \mathrm{i})$, true parameters set equal to estimates in Table 1 (top), and $\rho=0.30$

$\uparrow$ Ghosh ECB predictions computed by inserting MLEs of parameters

†LLEs; Other parameter estimates: $\hat{\theta}_{1}=-172.30, \hat{\theta}_{2}=0.23, \hat{\sigma}_{12}=-112073$, $\hat{\sigma}^{2}=510618, \hat{\rho}=0.29$

Figure 4: ECB Histograms Using Simulated Data from AR(1) Model (Table IV)
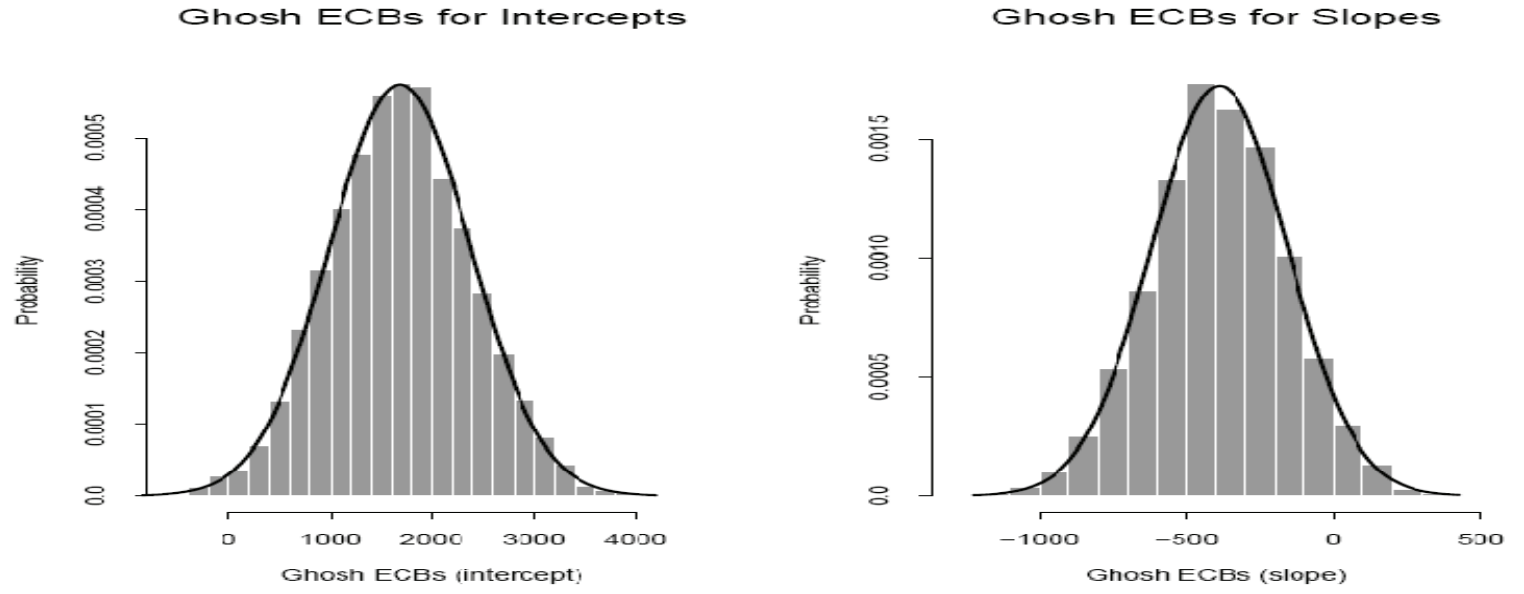


\section{LYLES, MOORE, MANATUNGA \& EASLEY}

The purpose of this article has been to outline and compare in detail the application of a direct (LX) CB approach considered by Lyles and Xu (1999) for certain mixed linear models, as opposed to the general method of Ghosh (1992). Both approaches were explored in the presence of covariates (possibly time-dependent), and it was concluded based on simulations and a realdata example that both may be effectively applied to achieve the moment-matching goals of the CB paradigm.

The LX approach, while presentable in closed form for the models considered herein, relies upon a strict form for candidate predictors and may not be straightforward to extend to arbitrary mixed linear models. However, as highlighted previously, the general method of Ghosh (1992) appears remarkably flexible and consistent in its application. In practice, it requires only EBLUPs and estimates of the posterior variances of the random effects being predicted, with the latter readily obtainable under normal-theory mixed models. It thus seems natural to compare the performance of the Ghosh method versus the LX approach in mixed model settings where the latter is available. The simulation studies summarized (and others, unreported) consistently show the Ghosh approach to be as effective as the direct LX method at matching moments, and also suggest slight prediction MSE gains via its use for unbalanced data.

Because the primary aim was to serve as proponents of the ECB approach under the mixed linear model, the results of the current study are encouraging. The CB paradigm of Ghosh (1992) relies on building blocks that are available in commercial software for mixed linear models (e.g., SAS PROC MIXED and similar procedures in other packages such as Splus, R, SPSS, STATA or BMDP). It was shown that it performs well relative to a direct, but far less flexible, CB approach developed expressly for mixed linear models. Although further assessments will be necessary, it is hoped that these results will encourage software developers to consider the possible inclusion of options to produce the Ghosh ECB predictions in future releases. This software advance would be welcome, for the purpose of allowing practitioners the freedom to select a validated alternative to the traditional EBLUP when overshrinkage could run counter to the objective at hand.

\section{Acknowledgements}

R. H. L. and A. K. M. were supported in part by an R01 from the National Institute of Environmental Health Sciences (ES012458). We thank the $\mathrm{P}^{2} \mathrm{C}^{2} \mathrm{HIV}$ study investigators and the National Heart, Lung and Blood Institute for use of their database, and appreciate support provided by the Biostatistics Core of the Emory Center for AIDS Research (P30 AI050409).

\section{References}

Diggle, P. J., Liang, K. Y., \& Zeger, S. L. (1994). Analysis of longitudinal data. NY: Oxford University Press.

Efron, B., \& Morris, C. (1971). Limiting the risk of Bayes and empirical Bayes estimators, part I: the Bayes case. Journal of the American Statistical Association, 66, 807-815.

Centers for Disease Control and Prevention. (1994). 1994 revised classification for human immunodeficiency virus infection in children less than 13 years of age. Morbidity and Mortality Weekly Report, 43, 1-10.

Ghosh, M. (1992). Constrained Bayes estimation with applications. Journal of the American Statistical Association, 87, 533-539.

Laird, N. M. \& Ware, J. H. (1982). Random-effects models for longitudinal data. Biometrics, 38, 963-974.

Littell, R. C., et al. (2006). SAS for mixed models, $2^{\text {nd }}$ Edition. Cary, NC: SAS Institute, Inc.

Louis, T. A. (1984). Estimating a population of parameter values using Bayes and empirical Bayes methods. Journal of the American Statistical Association, 79, 393-398.

Lyles, R. H. \& Xu, J. (1999). Classifying individuals based on predictors of random effects. Statistics in Medicine, 18, 3552.

Lyles, R. H., Manatunga, A. K., Moore, R. H., Bowman, F. D., \& Cook, C. (2007). Improving point predictions of random effects for subjects at high risk. Statistics in Medicine, $26,1285-1300$. 


\section{BAYES PREDICTIONS OF RANDOM INTERCEPTS AND SLOPES}

Moore, R.H., 2006. Prediction of random effects when data are subject to a detection limit. Unpublished Ph.D. dissertation, Department of Biostatistics, Emory University.

$\mathrm{P}^{2} \mathrm{C}^{2}$ HIV Study Group. (1996). The pediatric pulmonary and cardiovascular complications of vertically transmitted human immunodeficiency virus $\left(\mathrm{P}^{2} \mathrm{C}^{2} \mathrm{HIV}\right)$ infection study: Design and methods. Journal of Clinical Epidemiology, 49, 1285-1294.

SAS Institute, Inc. (2004a). SAS/STAT 9.1 user's guide. Cary, NC: SAS Institute, Inc.

SAS Institute, Inc. (2004b). SAS/IML 9.1 user's guide. Cary, NC: SAS Institute, Inc.

Searle, S.R., Casella, G., \& McCullogh, C.E. (1992). Variance components. NY: Wiley.

Shen, W., \& Louis, T.A. (1998). Triplegoal estimates in two-stage hierarchical models. Journal of the Royal Statistical Society Series B, 60, 455-471.

Stern, H.S., \& Cressie, N. (1999). Inference for extremes in disease mapping. In: A. Lawson, et al., (Eds). Disease mapping and risk assessment for public health, 63-84. Chichester: Wiley.

\section{Appendix}

A constrained Bayes predictor for the $i^{\text {th }}$ subject's random intercept $\left(\alpha_{\mathrm{i}}\right)$ may be obtained via calculations similar to those leading to $\widetilde{\beta}_{\mathrm{i}, \mathrm{LX}}$ in equation (8), as follows:

$$
\widetilde{\alpha}_{i, L X}=\tau_{i 1}+\tau_{i 2} \hat{\alpha}_{i, o l s}+\tau_{i 3} \hat{\beta}_{i, o l s},
$$

where

$$
\begin{gathered}
\tau_{\mathrm{i} 1}=\alpha\left(1-\tau_{\mathrm{i} 2}\right)-\beta \tau_{\mathrm{i} 3}, \\
\tau_{\mathrm{i} 2}= \pm\left[\sigma_{1}^{2} /\left\{\mathrm{v}_{\alpha \mathrm{i}}+\kappa_{\mathrm{i}}\left(2 \mathrm{c}_{\alpha \beta \mathrm{i}}+\kappa_{\mathrm{i}} \mathrm{v}_{\beta \mathrm{i}}\right)\right\}\right]^{1 / 2},
\end{gathered}
$$

and

with

$$
\tau_{\mathrm{i} 3}=\kappa_{\mathrm{i}} \tau_{\mathrm{i} 2},
$$

$$
\kappa_{\mathrm{i}}=\left(\mathrm{v}_{\alpha \mathrm{i}} \sigma_{12}-\sigma_{1}^{2} \mathrm{c}_{\alpha \beta \mathrm{i}}\right)\left(\mathrm{v}_{\beta \mathrm{i}} \sigma_{1}^{2}-\sigma_{12} \mathrm{c}_{\alpha \beta \mathrm{i}}\right)^{-1} .
$$

Specifically, $\widetilde{\alpha}_{i}$ defined in this way minimizes MSEP among predictors of the form (A1) subject to the constraints that $\mathrm{E}\left(\tilde{\alpha}_{\mathrm{i}}\right)=\mathrm{E}\left(\alpha_{\mathrm{i}}\right)=\alpha$ and $\operatorname{Var}\left(\tilde{\alpha}_{i}\right)=\operatorname{Var}\left(\alpha_{i}\right)=\sigma_{1}^{2}$, where the MSEP criterion is

$$
\begin{aligned}
& \mathrm{E}\left(\tilde{\alpha}_{\mathrm{i}}-\alpha_{\mathrm{i}}\right)^{2}= \\
& \left(\tau_{\mathrm{i} 2}^{2} \mathrm{v}_{\alpha \mathrm{i}}+\tau_{\mathrm{i} 3}^{2} \mathrm{~V}_{\beta \mathrm{i}}+2 \tau_{\mathrm{i} 2} \tau_{\mathrm{i} 3} \mathrm{c}_{\alpha \beta \mathrm{i}}\right)-2\left(\tau_{\mathrm{i} 2} \sigma_{1}^{2}+\tau_{\mathrm{i} 3} \sigma_{12}\right)+\sigma_{1}^{2}
\end{aligned}
$$

In an analogous manner, constrained Bayes predictor for $\mathrm{Y}_{\text {it }}^{*}$ is defined as

$$
\tilde{\mathrm{Y}}_{\mathrm{it}, \mathrm{LX}}^{*}=\varphi_{\mathrm{i} 1}+\varphi_{\mathrm{i} 2} \hat{\alpha}_{\mathrm{i}, \mathrm{ols}}+\varphi_{\mathrm{i} 3} \hat{\beta}_{\mathrm{i}, \mathrm{ols}} \text {, }
$$

where

$$
\begin{gathered}
\varphi_{\mathrm{i} 1}=\alpha\left(1-\varphi_{\mathrm{i} 2}\right)-\beta\left(\varphi_{\mathrm{i} 3}-\mathrm{t}_{\mathrm{i}}^{*}\right), \\
\varphi_{\mathrm{i} 2}= \pm\left[\psi_{\mathrm{i} 3} /\left\{\mathrm{v}_{\alpha \mathrm{i}}+\omega_{\mathrm{i}}\left(2 \mathrm{c}_{\alpha \beta \mathrm{i}}+\omega_{\mathrm{i}} \mathrm{v}_{\beta \mathrm{i}}\right)\right\}\right]^{1 / 2},
\end{gathered}
$$

and

$$
\varphi_{\mathrm{i} 3}=\omega_{\mathrm{i}} \varphi_{\mathrm{i} 2},
$$

with

$$
\begin{gathered}
\omega_{\mathrm{i}}=\left(\mathrm{v}_{\alpha \mathrm{i}} \psi_{\mathrm{i} 2}-\psi_{\mathrm{i} 1} \mathrm{c}_{\alpha \beta i}\right)\left(\mathrm{v}_{\beta \mathrm{i}} \psi_{\mathrm{i} 1}-\psi_{\mathrm{i} 2} \mathrm{c}_{\alpha \beta i}\right)^{-1}, \\
\psi_{\mathrm{i} 1}=\sigma_{1}^{2}+\mathrm{t}_{\mathrm{i}}^{*} \sigma_{12}, \psi_{\mathrm{i} 2}=\sigma_{12}+\mathrm{t}_{\mathrm{i}}^{*} \sigma_{2}^{2},
\end{gathered}
$$

and

$$
\psi_{i 3}=\sigma_{1}^{2}+t_{i}^{* 2} \sigma_{2}^{2}+2 t_{i}^{*} \sigma_{12} .
$$

This minimizes MSEP for predictors of the form (A3), subject to the constraints

$$
\mathrm{E}\left(\tilde{\mathrm{Y}}_{\mathrm{it}}^{*}\right)=\mathrm{E}\left(\mathrm{Y}_{\mathrm{it}}^{*}\right)=\alpha+\beta \mathrm{t}_{\mathrm{i}}^{*}
$$

and

$$
\operatorname{Var}\left(\widetilde{\mathrm{Y}}_{\mathrm{it}}^{*}\right)=\operatorname{Var}\left(\mathrm{Y}_{\mathrm{it}}^{*}\right)=\varphi_{\mathrm{i} 2}^{2} \mathrm{v}_{\alpha \mathrm{i}}+\varphi_{\mathrm{i} 3}^{2} \mathrm{v}_{\beta \mathrm{i}}+2 \varphi_{\mathrm{i} 2} \varphi_{\mathrm{i} 3} \mathrm{c}_{\alpha \beta \mathrm{i}}
$$

As with $\gamma_{\mathrm{i} 2}$ in equation (9), technically the choice of the positive or negative root to define $\tau_{\mathrm{i} 2}$ and $\varphi_{\mathrm{i} 2}$ should be based on which minimizes the corresponding MSEP criterion. However, it has been observed that the negative roots have never applied except in the case of $\gamma_{\mathrm{i} 2}$. 